PROCEEDINGS OF THE

AMERICAN MATHEMATICAL SOCIETY

Volume 128, Number 12, Pages 3761-3771

S 0002-9939(00)05479-4

Article electronically published on June 7, 2000

\title{
ALEXANDER NUMBERING OF KNOTTED SURFACE DIAGRAMS
}

\author{
J. SCOTT CARTER, SEIICHI KAMADA, AND MASAHICO SAITO
}

(Communicated by Ronald A. Fintushel)

\begin{abstract}
A generic projection of a knotted oriented surface in 4-space divides 3 -space into regions. The number of times (counted with sign) that a path from infinity to a given region intersects the projected surface is called the Alexander numbering of the region. The Alexander numbering is extended to branch and triple points of the projections. A formula that relates these indices is presented.
\end{abstract}

\section{INTRODUCTION}

An Alexander numbering of a classical knot diagram is depicted in Figure 1 In this figure, the planar regions that are separated by arcs of the curve are labeled with integers. The unbounded region is labeled 0 . The indices in two regions that are separated by an arc differ by 1 ; the region into which a normal arrow points has the larger index. Such numberings were used by J.W. Alexander [1] to give a combinatorial definition of his now well-known polynomial invariant (see also [18). An Alexander numbering for oriented knotted closed surfaces is defined similarly in Section 3 where indices are also assigned to the crossing points of a knotted surface diagram.

The title of this paper is an intentional pun. Alexander is also the given name of JSC's second son, who at age 8, looked at the diagram of Figure 2 (which is SK's diagram of the 3-twist spun trefoil and at the time was on the blackboard of JSC's office), and observed that the sum of the encircled labels added to 0 . Alexander Carter's observation on this diagram does not hold for every chart of knotted surfaces (see Figure 3), but there is a relation among the indices of the triple points and branch points that we develop herein. These diagrams represent surface braids, and will be explained in Section 2 .

1.1. Historical remarks. A formula between Whitney degree [27] and the Alexander numbering was given in [22], which was based on the integral calculus with respect to the Euler characteristic [26. The Alexander numbering for surfaces in 3 -space was used in [11] and [23]. Whitney's formula [28] relates the normal Euler

Received by the editors November 16, 1998 and, in revised form, March 1, 1999.

2000 Mathematics Subject Classification. Primary 57Q45; Secondary 57R20, 57R42.

Key words and phrases. Knotted surface diagrams, Alexander numbering, triple points, branch points, surface braids.

The second author is supported by a Fellowship from the Japan Society for the Promotion of Science.

(C)2000 American Mathematical Society 


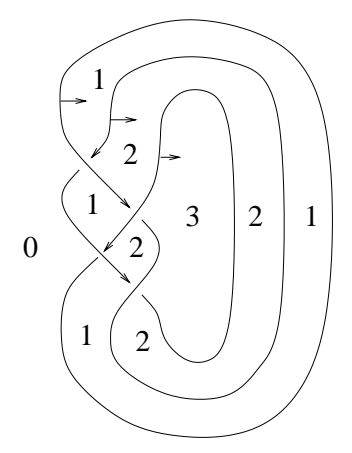

Figure 1. Alexander numbering of a classical knot

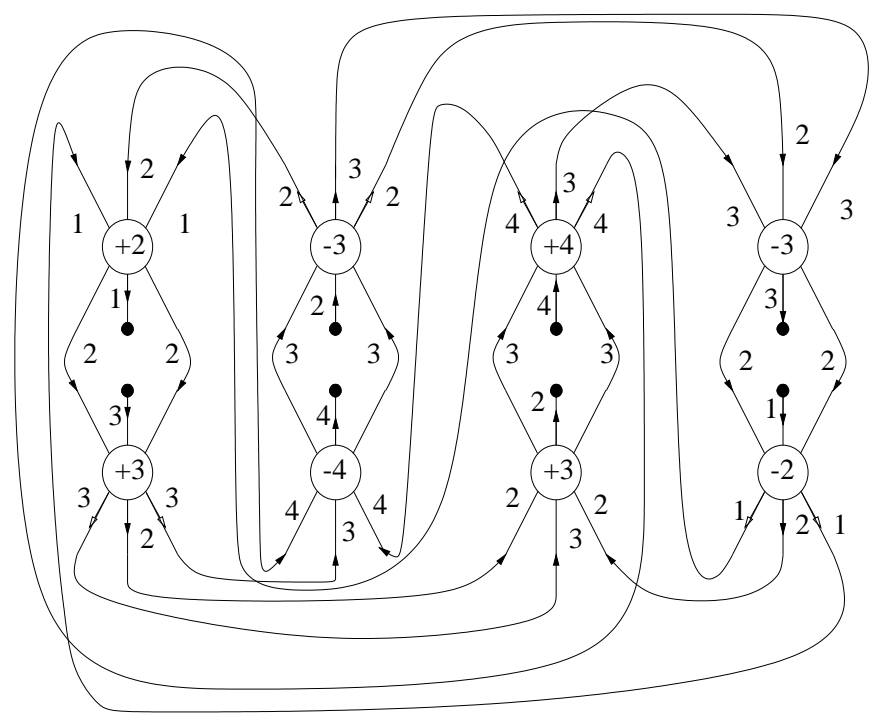

Figure 2. A braid chart of the 3-twist spun trefoil

class to the Euler characteristic (see also [5]). He conjectured that for non-orientable surfaces the number of achievable values is limited. This conjecture was proven by Massey in [21]; see 13] for a geometric proof. Banchoff relates the branch points to the normal Euler class in [3]. Banchoff's triple point formula [2] also relates singularities of surface maps to their intrinsic topology. Li shows [20] when a daisy graph is realizable as the multiple point set of a general position map. Generalizations of Banchoff's formula appear in [12] and [19]. Relations between branch points and triple points are found in [4] and [8]. A further generalization is presented in this paper.

Here is how the paper will be developed. Section 2 summarizes the notation also found in [9]. Section 3 defines the method of numbering. Section 4 contains the main results: Theorem 4.2 and its consequences. 


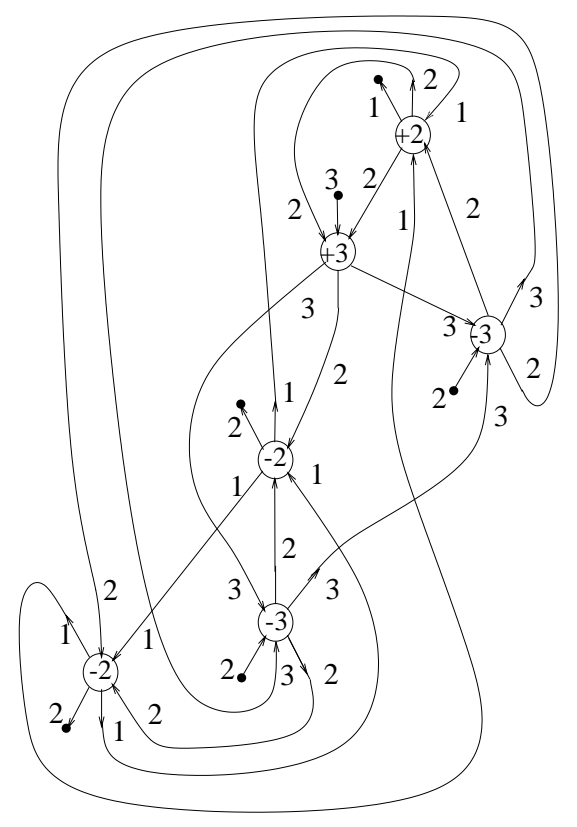

Figure 3. A braid chart of Fox's [10] Example 12

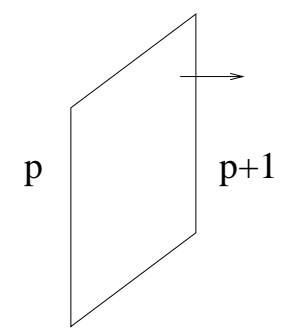

Figure 4. Alexander numbering changes by 1 through a sheet

\section{Notation}

In this section we review knotted surface diagrams and the chart description of surface braids that will be used in the paper. More details can be found in 9].

2.1. Generic projections and diagrams. For classical knots and links, underarcs are broken in the projections to define knot diagrams. We generalize this notion to knotted surfaces as broken surface diagrams. First we develop some notation. Let $f: F \rightarrow \mathbf{R}^{4}$ denote a smooth embedding of a closed surface $F$ into 4-dimensional space.

By deforming the map $f$ slightly by an ambient isotopy of $\mathbf{R}^{4}$, we may assume that $\pi \circ f$ is a general position map, where $\pi: \mathbf{R}^{4} \rightarrow \mathbf{R}^{3}$ is a projection onto a sub-affine-space which does not intersect $f(F)$. So any point of $(\pi \circ f)(F)$ has a neighborhood $U$ in 3 -space such that $(\pi \circ f)(F) \cap U$ looks like one of the pictures indicated in Figure 4 or the left of Figure 5 In other words, there is a diffeomorphism of $U$ into $\mathbf{R}^{3}$ such that the image of $F$ coincides with the intersection of 1,2 ( $a$ 

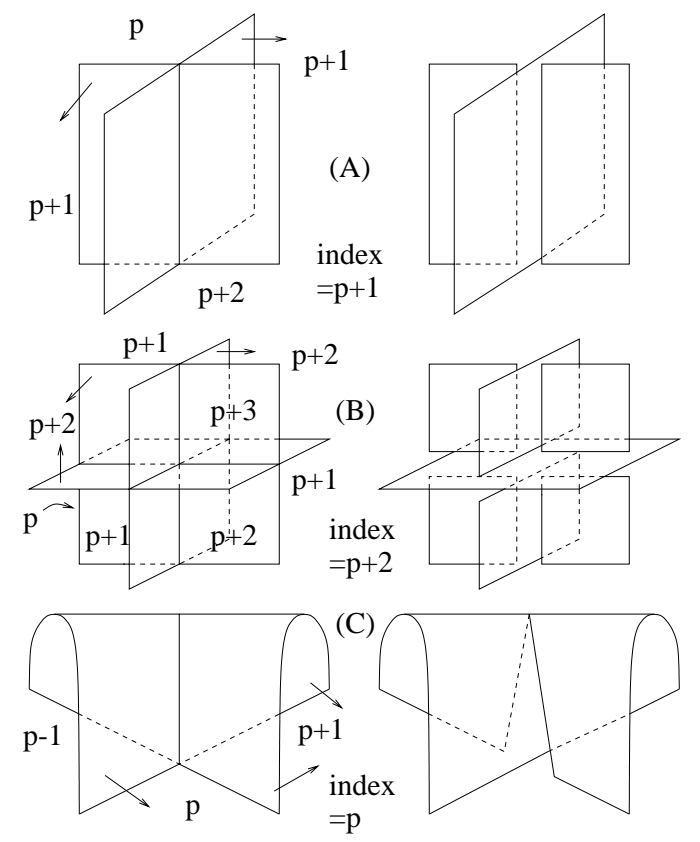

FIGURE 5. Projections, broken surface diagrams and indices

double point curve), or 3 coordinate planes (a triple point), or is like the cone on a figure 8 (a branch point). The neighborhood of a double point curve, a triple point, or a branch point is depicted in Figure 5 (A), (B), or (C), respectively. When the surface is oriented, we take normal vectors $\vec{n}$ to the projection of the surface such that the triple $\left(\vec{v}_{1}, \vec{v}_{2}, \vec{n}\right)$ matches the orientation of 3 -space, where $\left(\vec{v}_{1}, \vec{v}_{2}\right)$ defines the orientation of the surface. Such normal vectors are defined on the projection at all points other than the isolated branch points.

There is an immersion in $\mathbf{R}^{3}$ of a compact 1-manifold with boundary whose image is the closure of the set

$$
\left\{x \in \pi(f(F)) \mid \pi\left(f\left(x_{1}\right)\right)=x=\pi\left(f\left(x_{2}\right)\right) \text { for some } x_{1} \neq x_{2}, \quad \text { where } x_{1}, x_{2} \in F\right\} .
$$

The closure of the above set is called the double point set. The image of the boundary points of the 1-manifold are the branch points of the map $\pi \circ f$. At a triple point three curves meet transversely, so a triple point is a 6 -valent vertex.

In the diagram of a knotted surface, one of the two sheets is broken along the double point arcs to indicate that it is higher than the other sheet in the projection direction of $\pi$. Broken surfaces are indicated in Figure 5 .

The double point curves are oriented in such a way that the orientation $\vec{v}$ together with normal vectors of top and bottom sheets $\left(\overrightarrow{n_{1}}, \overrightarrow{n_{2}}, \vec{v}\right)$ matches the orientation of space. The double point curve depicted in Figure 5(A) is oriented downward.

2.2. Signs of branch and triple points. The sign of a triple point is defined as follows 8 . For the normal vectors $v_{1}, v_{2}, v_{3}$ of top, middle, bottom sheet, respectively, if the triple $\left(v_{1}, v_{2}, v_{3}\right)$ matches the orientation of $\mathbf{R}^{3}$, then the sign is positive, and negative if otherwise. 
The sign of a branch point is defined as follows [4, 5]. If the the double curve ending at the given branch point is oriented towards (resp. away from) the branch point, then the sign is negative (resp. positive). The triple point depicted in Figure 5 is negative, and the branch point is positive.

2.3. Charts of surface braids. The notion of surface braids, a generalization of Artin's braid theory, was originally proposed by Viro, and a similar notion had been used by Rudolph [25]. We review the chart description of surface braids as developed in [6, 7, 14, 15].

Figure 6 shows how to express certain generic surfaces in 3 -space by means of planar graphs. We consider a surface $S$ (this $S$ corresponds to $f(F)$ in the preceding section) in a box $B=I_{1} \times I_{2} \times I_{3} \subset \mathbf{R}^{3}$ a schematic of which is depicted on the right of Figure 6, where $I_{j}$ denotes a copy of the unit interval, for $j=1,2,3$. We require that the surface $S$ in $B$ satisfies the following conditions.

- $S$ is generic.

- The boundary $\partial S$ of $S$ is a closed trivial braid contained in $\partial\left(I_{1} \times I_{2}\right) \times I_{3}$.

- The projection $\pi_{1}: I_{1} \times I_{2} \times I_{3} \rightarrow I_{1} \times I_{2}$ restricted to $S$ is a branched covering such that each branch point is simple. The preimage of a branch point, then, consists of a unique branch point of degree 2 and a collection of points around which the projection is a local homeomorphism.

Let $D$ be the double point set of $S$. Then $\pi_{1}(D) \subset I_{1} \times I_{2}$ is a planar graph. The graph $\pi_{1}(D)$ has univalent vertices corresponding to branch points, 4 -valent vertices that correspond to the crossings of the projections of double arcs, and 6-valent vertices corresponding to triple points of $S$. A generic intersection $S \cap I_{1} \times\{t\} \times I_{3}$ consists of intersecting strings in $I_{1} \times\{t\} \times I_{3}$. Such intersections are shown in the figure by movie strips.

When the surface $S$ is the projection of a surface braid in the 4-disk $I_{1} \times I_{2} \times$ $I_{3} \times I_{4}$, the fourth coordinate can be indicated in the movies by broken arcs. Then the arcs in the graph $\pi_{1}(D)$ are oriented to agree with the orientation of the corresponding double curves. A downward pointing arrow in the chart (with respect to the height direction in the page) that is labeled with an integer $i$ corresponds to the $i$ th braid generator in which the $i$ th string passes over the $(i+1)$ st string. The graph $\pi_{1}(D)$ with this orientation is called a braid chart [15].

The univalent vertices of a chart correspond to branch points and are called black vertices. The 6 -valent vertices correspond to triple points and are called white vertices. The signs of triple points on a braid chart can be determined as follows: if the indices of arcs at a triple point are $p$ and $p+1$ and the middle incoming arc is labeled by $p+1$, then the triple point (white vertex) is positive, and otherwise negative.

By capping off the nested boundary circles on the boundary of the box $B$ by nested disjoint disks, we get a closed surface in 3 -space. If it is a projection of a knotted surface, taking such a closure gives rise to a closed surface braid in 4-space.

Figures 2 and 3 represent the braid charts of 3-twist-spun trefoil (see 24] for example) and Fox's Example 12 in [10, respectively.

\section{Alexander numberings}

Let $F$ denote an oriented surface that is embedded in 4-space via a map $f: F \rightarrow$ $\mathbf{R}^{4}$. Consider a general position projection of the knotting $f$ into 3 -space and the associated knotted surface diagram. Choose a normal orientation in 3-space for 

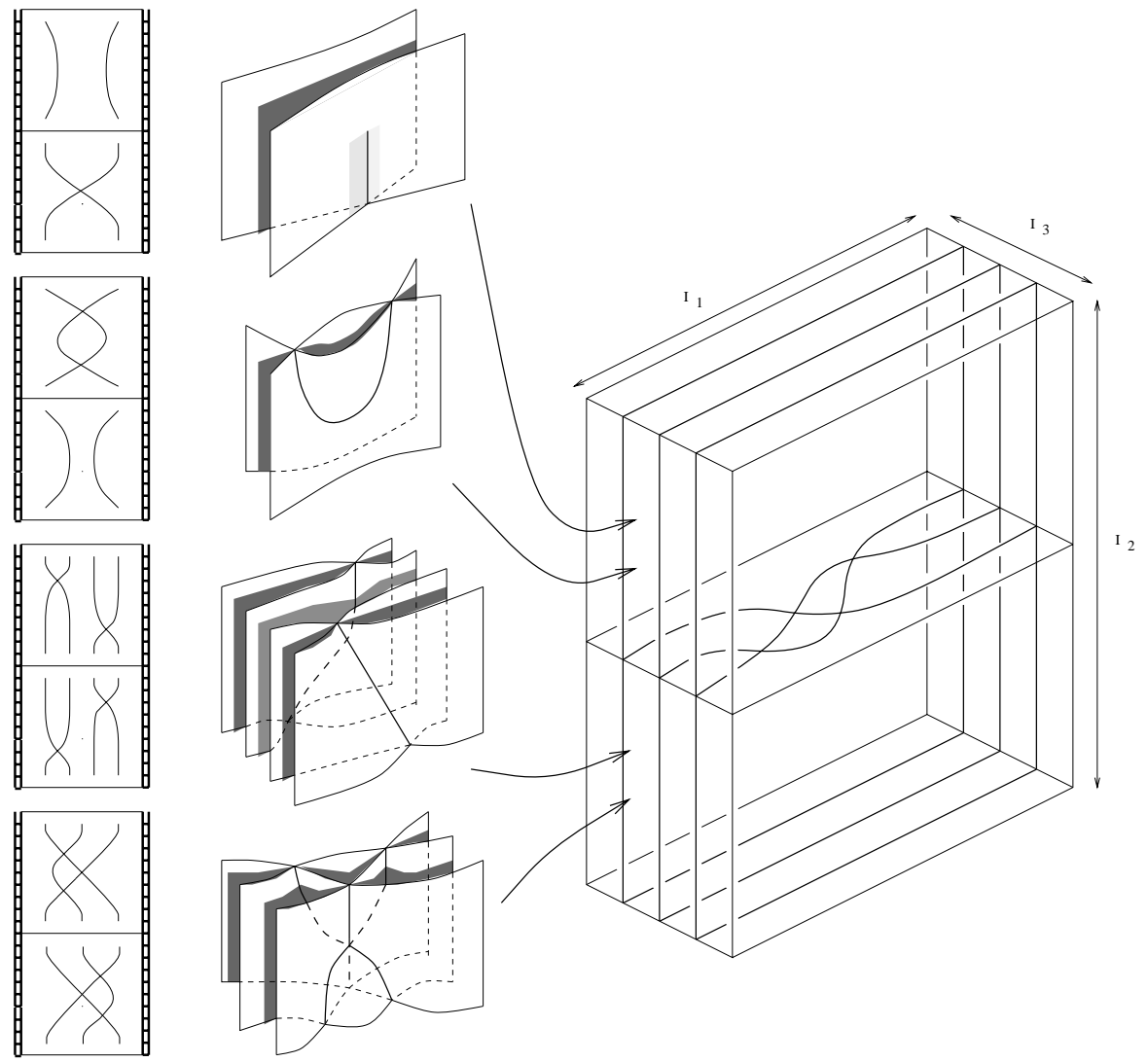

Figure 6. Generic surfaces, movies, and surface braids

the projection such that the tangent orientation followed by the normal orientation agrees with the right-handed orientation of 3-space. Number with integers the 3dimensional regions in the complement of the projection according to the convention that (a) the unbounded region is numbered 0 , (b) regions that are separated by a 2-dimensional face are numbered consecutively, and (c) the normal vector to the surface points towards the region with largest number. Such an indexing is called an Alexander numbering. This was defined in 11, 23. and was called index or degree.

If the regions adjacent to a double arc are numbered $p, p+1, p+1$, and $p+2$, then the Alexander number of the double arc is $p+1$ (Figure 5(A)). In a neighborhood of a triple point, one of the adjacent regions is numbered $p$, three are numbered $p+1$, three are numbered $p+2$, and one is numbered $p+3$. The Alexander number of the triple point is $p+2$ (Figure 5 (B)). The Alexander number of a branch point is the number of the region that is not interior to the figure 8 . Thus if the regions interior to the figure 8 are labeled $p-1$ and $p+1$, then the Alexander number of the branch point is $p$ (Figure $5(\mathrm{C})$ ).

When a knotted surface is given as a surface braid, then the cross sectional braids are closed with arcs that pass to the right as in Figure 11. We may assume that the surface is oriented so that the normal direction points consistently to the right 

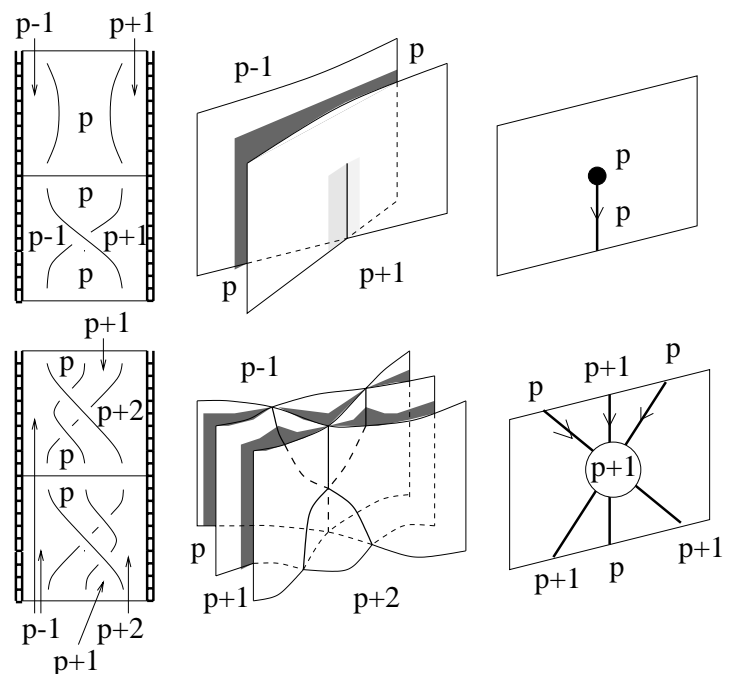

FIGURE 7. Indices at braid generators

on the "braid portion of the diagram" (on the closure part, the normal points left: Figure 1). The braid index of a branch point is the index of the braid generator that is created or destroyed at the black branch point in the chart. The braid index of a triple point is the largest braid index among the indices that appear adjacent to the triple point. Thus at a white vertex in a braid chart 3 edges each are incident with indices $p$ and $p+1$. The braid index of the vertex is $p+1$. A white vertex in the chart corresponds to the braid relation $\sigma_{p} \sigma_{p+1} \sigma_{p}=\sigma_{p+1} \sigma_{p} \sigma_{p+1}$; the braid index is $(p+1)$. The situation is depicted in Figure 7

3.1. Observation. The Alexander numberings of the double, branch, and triple points agree with the braid index of the corresponding points when the surface is in braid form.

Proof. The region containing $\infty$ is immediately to the left of the first arc in a braid word.

The Alexander numberings of double, branch, triple points are indicated in Figures 2 and 3 in their charts.

\section{Statement of MAin RESUlt}

Definition 4.1. Let $T(p, \delta)$ denote the number of triple points of Alexander index $p$ with sign $\delta= \pm$. Let $B(p, \sigma)$ denote the number of branch points of Alexander index $p$ with sign $\sigma= \pm$.

Theorem 4.2. For a diagram of a knotted oriented closed surface,

$$
\sum_{p, \sigma} \sigma x_{p} B(p, \sigma)+\sum_{q, \delta} \delta y_{q} T(q, \delta)=0
$$

provided $y_{p}=x_{p}-x_{p-1}$. 
Proof. Consider an arc of double points of index $p$. Such an arc can start at a branch point of index $p$ or start at a triple point of index $p$ or index $(p+1)$. There are two double arcs of index $p$ that start at each positive triple point of index $p$ and each negative triple of index $(p+1)$. One such arc starts at a negative triple point of index $p$, positive triple of index $(p+1)$, or branch point of index $p$. Thus the number of edges of index $p$ is

$$
E(p)=B(p,+)+2 T(p,+)+T(p,-)+T(p+1,+)+2 T(p+1,-) .
$$

The ending points of such arcs can be determined similarly. So,

$$
E(p)=B(p,-)+2 T(p,-)+T(p,+)+T(p+1,-)+2 T(p+1,+) .
$$

We obtain

$$
B(p,+)-B(p,-)=T(p+1,+)-T(p+1,-)-(T(p,+)-T(p,-)) .
$$

We call this relation equation $(\star)$. Multiply through by $x_{p}$, and sum over all $p$ to get:

$$
\begin{aligned}
\sum_{p, \sigma} \sigma x_{p} B(p, \sigma) & =\sum_{p, \delta} \delta x_{p} T(p+1, \delta)-\sum_{p, \delta} \delta x_{p} T(p, \delta) \\
& =\sum_{p, \delta} \delta\left(x_{p-1}-x_{p}\right) T(p, \delta)
\end{aligned}
$$

This completes the proof.

Corollary 4.3. If $y_{p}=0$ for all $p$, then $x_{p}=x_{p+1}$. Therefore,

$$
\sum_{p, \sigma} \sigma B(p, \sigma)=0
$$

So we recover the fact that oriented surfaces have trivial normal Euler classes because the number of signed branch points add up to the normal Euler number [5].

Corollary 4.4. If $y_{p}=p$, and $x_{1}=1$, then $x_{p}=p(p+1) / 2$, and

$$
\sum_{p, \sigma} \sigma \frac{p(p+1)}{2} B(p, \sigma)+\sum_{q, \delta} \delta q T(q, \delta)=0 .
$$

Corollary 4.5. If $y_{p}=1$, and $x_{1}=1$, then $x_{p}=3 p$, and

$$
\sum_{p, \sigma} \sigma p B(p, \sigma)+\sum_{q, \delta} \delta T(q, \delta)=0
$$

Corollary 4.6. If $B(p, \sigma)=0$ for all $p$, then $T(p,+)=T(p,-)$. In particular, this is true if the projection of the surface is immersed.

Corollary 4.7. $T(p,+)-T(p,-)=\sum_{i<p} B(i,+)-B(i,-)=-\sum_{i \geq p} B(i,+)-$ $B(i,-)$.

Proof. This is a consequence of equation $\star$. Observe that these sums are finite.

Theorem 4.8. There exists a diagram of an unknot or unlink that realizes prescribed values for $B(p, \pm)$ and $T(p, \pm)$ that satisfy the formula in Theorem 4.2 


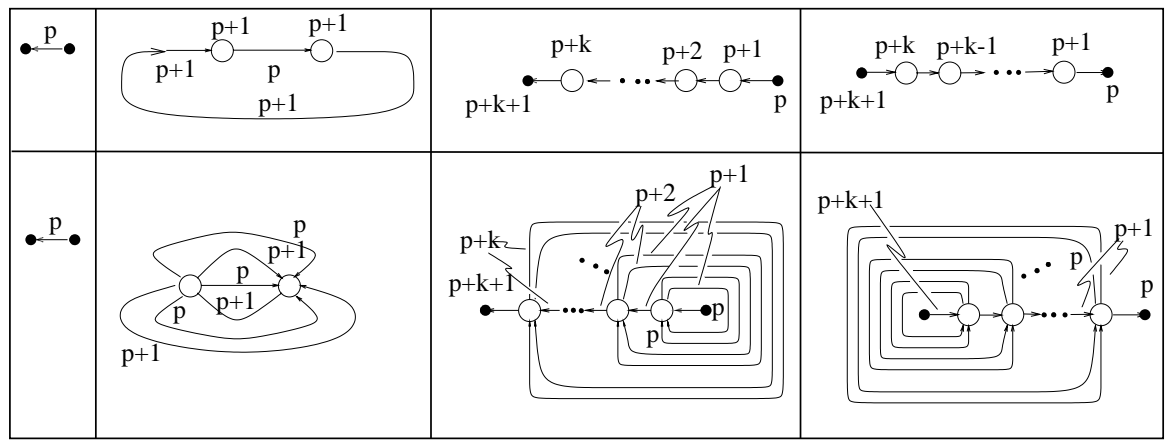

Figure 8. Some charts of unknotted surfaces

Proof. By adding nested spheres near $\infty$, we may assume that the labels are all positive. We construct a labeled planar graph with the correct number of black and white vertices, and then we use this to construct a chart of an unlink. Between lines $x=p$ and $x=p+1$ put $B(p,+)$ (resp. $B(p,-))$ black vertices with horizontal arrows that point away from (resp. toward) the vertex. Put $T(p+1,+)$ (resp. $T(p+1,-)$ ) white vertices in the same region with horizontal right-pointing (resp. left-pointing) incoming (resp. outgoing) arrows labeled $p$ and right-pointing (resp. left pointing) outgoing (resp. incoming) arrows labeled $(p+1)$. Along the line $x=p$ stack $|T(p,+)-T(p,-)|$ arrows that point to the right if $T(p,+)-T(p,-)>0$ arrows and to the left otherwise. Connect the arrows according to the rules that (1) lines only cross the line $x=p$ along the stacked arrows, (2) each possible pair of white vertices are connected in a loop, (3) a pair of black vertices are linearly chained by a (possibly empty) set of white vertices. The arcs in the graph may intersect. The only loops in the graph connect a pair of white vertices.

Embed the graph in the plane. Figure 8 indicates how to replace the graph with bits of charts. The resulting surface can be seen to be unknotted by means of chart moves. See [9, 14] for a description of the moves. This completes the proof.

4.9. Oriented surfaces with singular points. In this section we generalize Theorem 4.2 to generically immersed oriented surfaces in 4-space. Such surfaces have isolated transverse double points that we call singular points to distinguish them from arcs of double points in the projection. These can be indicated by broken surface diagrams as indicated in Figure 9 . The sign of such a singular point is negative if the oriented double arcs both point toward the singular point. The sign is positive if the double arcs point away from the singular point. Say that two of the regions adjacent to the singular point have indices $(p+1)$; the other two have indices $p$ and $p+2$. Then the index of singular point is $(p+1)$.

Let $D(p, \epsilon)$ denote the number of singular points of index $p$ and sign $\epsilon$.

Theorem 4.10. For a diagram of a knotted oriented closed surface with singular points,

$$
\sum_{p, \sigma} \sigma x_{p} B(p, \sigma)+\sum_{q, \delta} \delta y_{q} T(q, \delta)+\sum_{r, \epsilon} \epsilon z_{r} D(r, \epsilon)=0
$$

provided that $y_{p}=x_{p}-x_{p-1}$ and $z_{p}=2 x_{p}$. 


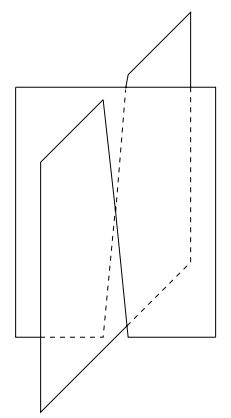

Figure 9. A broken surface diagram at a singular point

Proof. By counting edges of label $p$, as before, we obtain

$$
\begin{gathered}
E(p)=B(p,+)+2 T(p,+)+T(p,-)+T(p+1,+)+2 T(p+1,-)+2 D(p,+) \\
=B(p,-)+2 T(p,-)+T(p,+)+T(p+1,-)+2 T(p+1,+)+2 D(p,-) .
\end{gathered}
$$

Then

$$
\begin{gathered}
B(p,+)-B(p,-)+2(D(p,+)-D(p,-)) \\
=T(p+1,+)-T(p+1,-)-(T(p,+)-T(p,-)) .
\end{gathered}
$$

Multiply through by $x_{p}$, and sum over all $p$ to obtain the result.

\section{REFERENCES}

[1] Alexander, J.W., A lemma on systems of knotted curves, Proc. Nat. Acad. Science USA, 9 (1923), 93-95.

[2] Banchoff, T.F., Triple points and surgery of immersed surfaces, Proc. AMS 46, No.3 (Dec. 1974), 403-413. MR 51:14065, MR 51:14066

[3] Banchoff, T.F., Double tangency theorems for pairs of submanifolds, in Geometry Symposium Utrecht 1980 ed. Looijenga, Seirsma, and Takens, LNM v. 894, Springer-Verlag (1981), 26-48. MR 83h:53005

[4] Carter, J.S., Carrara V., and Saito, M. Singularities of the projections of surfaces in 4-space, Preprint.

[5] Carter, J.S., and Saito, M., Canceling branch points on the projections of knotted surfaces in 4-space, Proc. of the AMS. 116, No 1 (Sept 1992), 229-237. MR 93i:57029

[6] Carter, J.S.; Saito, M., Braids and movies. J. Knot Theory Ramifications 5 (1996), no. 5, 589-608. MR 97j:57028

[7] Carter, J.S.; Saito, M., Knotted surfaces, braid moves, and beyond. Knots and quantum gravity (Riverside, CA, 1993), 191-229, Oxford Lecture Ser. Math. Appl., 1, Oxford Univ. Press, New York, 1994. MR 95m:57036

[8] Carter, J.S. and Saito, M. Normal Euler classes of knotted surfaces and triple points on projections, Proc. Amer. Math. Soc. 125 (1997), no. 2, 617-623. MR 97d:57030

[9] Carter, J.S. and Saito, M., Knotted surfaces and their diagrams, Surveys and monographs, vol. 55, A.M.S., Jan. 1998. MR 98m:57027

[10] Fox, R.H., A quick trip through knot theory. in Cantrell and Edwards, "Topology of Manifolds," Prentice Hall (1962). MR 25:3522

[11] Goryunov, V. Local invariants of mappings of surfaces into three-space, The Arnold-Gelfand mathematical seminars, 223-255, Birkheuser Boston, Boston, MA, 1997. [MR 97m:57040]

[12] Izumiya, H. and Marar, W.L. The Euler Characteristic of a generic wavefront in a 3manifold, Proc. AMS 118 No 4 (August 1993), 1347-1350. MR 93j:58014 
[13] Kamada, S., Nonorientable surfaces in 4-space, Osaka J. Math. 26 (1989), no. 2, 367-385. MR 91g:57022

[14] Kamada, S., Surfaces in $\mathbf{R}^{4}$ of braid index three are ribbon, J. Knot Theory Ramifications 1 (1992), no. 2, 137-160. MR 93h:57039

[15] Kamada, S., 2-dimensional braids and chart descriptions, "Topics in Knot Theory (Erzurum, 1992)," 277-287, NATO Adv. Sci. Inst. Ser. C Math. Phys. Sci., 399, Kluwer Acad. Publ., (Dordrecht, 1993). CMP 94:07

[16] Kamada, S., A characterization of groups of closed orientable surfaces in 4-space. Topology 33 (1994), no. 1, 113-122. MR 95a:57002

[17] Kamada, S., Alexander's and Markov's theorems in dimension four, Bull. Amer. Math. Soc. (N.S.) 31 (1994), no. 1, 64-67. MR 94j:57023

[18] Kauffman, L.H., "On Knots." Ann. of Math. Studies, Princeton U. Press, 1987. MR 89c: 57005

[19] Ko, K.H. and Carter, J.S., Triple points of immersed surfaces in three dimensional manifolds, Topology and Its Applications 32, (1989), 149-159. MR 90i:57018

[20] Li, G.S., On self-intersections of immersed surfaces, Proc. Amer. Math. Soc. 126 (1998), pp. 3721-3726. MR 99b:57045

[21] Massey, W.S., Proof of a conjecture of Whitney, Pacific J. Math. 31 (1969), 143-156. MR 40:3570

[22] McIntyre, M.; Cairns, G., A new formula for winding number, Geom. Dedicata, 46 (1993), 149-160. MR 94k:55005

[23] Mikhalkin, G.; Polyak, M. Whitney formula in higher dimensions, J. Differential Geom. 44 (1996), no. 3, 583-594. MR 97m:57043

[24] Rolfsen, D., Knots and Links, Publish or Perish Press, Berkeley, 1976. MR 58:24236]

[25] Rudolph, L., Braided surfaces and Seifert ribbons for closed braids. Comment. Math. Helv. 58 (1983), no. 1, 1-37. MR 84j:57006

[26] Viro, O.Ya., Some integral calculus based on Euler characteristic, Lect. Notes Math., 1346 (1988), 127-138. MR 90a:57029]

[27] Whitney, H., On regular closed curves in the plane, Compositio Math. 4 (1937), 276-284.

[28] Whitney, H., On the topology of differentiable manifolds, in Lectures in Topology, (Wilder and Ayres, eds.), University of Michigan Press (1941), 101-141. MR 3:133a

Department of Mathematics, University of South Alabama, Mobile, Alabama 36688

E-mail address: carter@mathstat.usouthal.edu

Department of Mathematics, Osaka City University, Osaka 558-8585, Japan

E-mail address: kamada@sci.osaka-cu.ac.jp

Current address: University of South Alabama, Mobile, Alabama 36688

Department of Mathematics, University of South Florida, Tampa, Florida 33620

E-mail address: saito@math.usf.edu 